\section{Pulmonary Aspergilloma}

From time to time spores of the fungus aspergillus are inevitably inhaled into the lungs from the atmosphere. Occasionally they stimulate the formation of antibodies in the serum of healthy persons. When the lungs are chronically damaged, for example by sarcoidosis or pneumoconiosis, the bronchi and surrounding tissue may become inflamed. In debilitating states such as cancer, especially after immunosuppressive therapy, an extensive necrotizing bronchopneumonia may develop, or haemorrhagic infarcts may follow penetration of the vasculature. Allergic persons may become sensitive to aspergillus, with the development of asthmatic attacks or eosinophilic pulmonary infiltrations. The fungus seems to have a special predilection for pulmonary cavities, such as occur in tuberculosis, chronic lung abscess, bronchiectasis, bullae, and cysts, in which it multiplies to form an aspergilloma (mycetoma) or fungus ball.

The diagnosis of an aspergilloma is radiological in the first instance. An irregularly rounded opacity is seen surrounded, at least in part, by a thin halo of air. Sometimes a mycetoma can be strongly suspected when such an opacity develops without a halo in a previously observed cavity. Confirmation of the diagnosis rests principally on the detection of precipitating antibodies in the serum. ${ }^{12}$ Agar-gel double diffusion tests show a wide range of antibodies against antigens prepared from cultures of Aspergillus fumigatus, with the development of up to 20 diffusion arcs. The degree of positivity is graded according to whether $2-4$, $5-7$, or 8 or more arcs are visible. J. L. Longbottom and colleagues $^{1}$ found positive reactions in 57 of 58 patients with an aspergilloma, in 56 against $A$. fumigatus, and in one against $A$. nidulans.

During 1964-5 the British Tuberculosis Association ${ }^{3}$ collected data from 55 chest clinics on 544 patients having persistent cavities measuring at least $2.5 \mathrm{~cm}$ in diameter and in whom sputum cultures of Mycobacterium tuberculosis had been positive but were negative for the previous year, so-called open healed tuberculous cavities. Of these, 59 had radiological evidence of an intracavitary fungus ball and another 56 had a positive precipitin test without evidence of a mycetoma. The patients were reassessed in 1967-8. ${ }^{4}$ During the interval $18 \%$ had died, the mortality rate being similar for those with and without a mycetoma. During the three years the percentage of patients with a mycetoma rose from 11 to 17 , and with a positive precipitin test without mycetoma from 10 to 14 . From the two surveys together several conclusions were drawn. Patients with a positive precipitin test but without a mycetoma at the first interview were twice as likely to develop a mycetoma as the remainder. Mycetomata developed as frequently in relatively new cavities as in those of long standing, but the determining factor was probably the state of activity of the tuberculosis, since fungus balls rarely develop in active tuberculous cavities. A few mycetomata disappeared spontaneously or during a pyogenic infection of the cavity. When the mycetoma was resected, the precipitin test became weaker but was rarely negative.

A positive precipitin test is almost invariably found when an aspergilloma exists, and it forms the basis of diagnosis, since the fungus is unlikely to be isolated from the sputum of more than about one-third of patients. Some difficulty may be experienced in interpreting a positive result when no aspergilloma is demonstrable. In this series the total posi- tives rose from 25 to $34 \%$ of patients over the three years. Some undoubtedly had allergy to aspergillus with positive skin tests (which are often negative in aspergilloma). Others had weakening of the reaction over the three years, suggesting a temporary infection of the damaged lungs without recognizable aspergilloma or, when a fungus ball existed, death of the fungus.

The treatment of pulmonary aspergilloma is most unsatisfactory. Many antifungal remedies have been tried by various routes-for example, brilliant green, pimaricin, and amphotericin B-but without measurable success. Fortunately the ball seems to have comparatively little effect on general health or the prognosis of the underlying disease. It may stimulate fibrosis locally and cause a troublesome cough. ${ }^{4}$ Occasionally recurrent and severe haemoptyses may be ascribed to it, in which case surgical resection should be seriously considered.

Fungal invasion of the lungs is fairly rare. Aspergilli and other moulds are widespread in the atmosphere and they multiply freely in the air passages when the conditions are favourable for them. The increasing usage of antibacterial and immunosuppressant drugs may facilitate the development of pulmonary mycoses, a clear example of which is provided by the formation of aspergillomata in open healed tuberculous cavities.

1 Longbottom, J. L., Pepys, J., and Clive, F. T., Lancet, 1964, 1, 588. Longbottom, J. L., and Pepys, J., journal of Pathology and Bacteriology, 1964, 88, 141 .

3 Report from the Research Committee of the British Tuberculosis Association, Tubercle, $1968,49,1$.

Report from the Research Committse of the British Thoracic and Tuberculosis Association, Tubercle, 1970, 51, 227.

\section{Aberystwyth Meeting}

The University College of Wales provided a self-contained setting for the B.M.A.'s fourteenth Annual Clinical Meeting. The proceedings, which opened with a lecture on "The Flight From Reason" by Professor Michael Swann, are fully reported on p. 147 of this issue. Most of the visitors stayed in the halls of residence, and indeed until the last evening the formal scientific and social events were confined to the university campus, which encouraged a high attendance at all the sessions.

Whatever their motives for attending, the audiences were not disappointed. Lively discussion after the symposia and many of the shorter meetings paid tribute to the quality of the lectures. Much of the credit for this undoubted success must go to the local organizing committee. Dr. Hugh Herbert, the chairman, and Dr. W. H. Beasley, the honorary secretary, showed their imaginative approach at the start of the meeting when young girls in national costume waited on the guests at the reception in the University Great Hall given jointly by the University and the Aberystwyth Division. Though cold, the weather was mostly bright, and the Ladies' Committee under Mrs. H. Herbert and Mrs. Rowland Edwards offered a fine range of tours of the imposing local countryside and was also responsible for some very attractive flower arrangements.

Only at the end of the meeting, then, did most of the medical visitors descend the hill into Aberystwyth, where they were warmly welcomed by the Mayor and Corporation at the King's Hall. Aberystwyth can certainly be assured that the B.M.A. enjoyed its stay. 